\title{
Clinical utility of TSH receptor antibody levels in Graves' orbitopathy: a comparison of two TSH receptor antibody immunoassays
}

\author{
GABRIELA A. BLUSZCZI, TOMASZ BEDNARCZUK ${ }^{1}$, ZBIGNIEW BARTOSZEWICZ ${ }^{1}$, \\ AGNIESZKA KONDRACKA ${ }^{l}$, KLAUDIA WALCZAK ${ }^{1}$, ZUZANNA ŻURECKA', \\ URSZULA DEMKOW', PIOTR MIŚKIEWICZ ${ }^{1}$
}

${ }^{1}$ Department of Internal Medicine and Endocrinology, Medical University of Warsaw, Warsaw, Poland

${ }^{2}$ Department of Laboratory Diagnostics and Clinical Immunology of Developmental Age, Medical University of Warsaw, Warsaw, Poland

\begin{abstract}
Introduction: Thyroid stimulating hormone (TSH) receptor antibodies (TRAB) play a role in the development of Graves' orbitopathy (GO), and measurements of the TRAB level may be helpful in monitoring GO treatment.

Aim of the study: To assess the correlation of TRAB levels measured with two different assays: third-generation TRAB assay (TRAB Cobas) and novel Immulite assay (TRAB Immulite), in patients with moderate-to-severe GO treated with intravenous glucocorticoid pulse therapy (ivGCs).

Material and methods: Forty patients with active, moderate-to-severe GO underwent clinical and laboratory evaluation before, in the middle, and after ivGCs therapy. The correlation of TRAB levels with GO signs was evaluated. Laboratory and clinical findings were compared according to the response to ivGCs. TRAB concentration was measured with Immulite TSI assay and with Elecsys IMA.

Results: All patients were TRAB positive in both assays at the beginning of the treatment. The decrease of both TRAB Immulite and Cobas levels in serum during ivGCs was statistically significant. We observed strong correlation between both TRAB levels before and after ivGCs. There was no statistically significant difference in antibody levels between patients with good response and no response to the treatment. We did not find any correlation between antibody levels and GO features before the therapy, but measurements during ivGCs showed comparable correlation of both TRAB levels with GO activity.

Conclusions: We found similarity between Immulite assay and third-generation TRAB assay in the assessment of patients with GO treated with ivGCs. Both TRAB levels showed comparable correlation with $G O$ activity during ivGCs therapy.
\end{abstract}

Key words: Graves' disease, Graves' orbitopathy, intravenous glucocorticoids, TSH receptor antibodies, automated immunoassay.

(Centr Eur J Immunol 2018; 43 (4): 405-412)

\section{Introduction}

Graves' orbitopathy (GO) is the most common extrathyroidal manifestation of Graves' disease (GD) [1, 2]. Active, moderate-to-severe form of GO represents about $5 \%$ of cases [3] and requires intravenous glucocorticoid pulse therapy (ivGCs) [2]. Unfortunately, the efficacy of the treatment and patient satisfaction is lower than expected $[4,5]$.

Although some established risk factors help to predict GO development and severity [2], there are no adequate data on prognostic markers that could be used to improve GO management. Previous studies showed the potential role of thyroid stimulating hormone (TSH) receptor antibodies (TRAB) in the pathogenesis of GO and hence the possibility of including TRAB measurements in the GO assessment and monitoring of its treatment [6-8].

In general, TRAB measurements can be performed using either immunoassays (IMAs) or bioassays. Second are cell-based tests that assess the functional activity of TRAB; however, their usage is limited to experienced laboratories only [9]. IMAs were introduced in the 1980s, and since then their technology has been largely improved. [10]. The first-generation of TRAB competitive assays, using porcine cells and bovine labelled TSH as a competitor, were characterised by low sensitivity [11]. The second-generation assays quantitatively measuring TRAB against the recombinant human TSH receptors (TSH-R) were more

Correspondence: Piotr Miśkiewicz, PhD, Department of Internal Medicine and Endocrinology, Medical University of Warsaw,

61 Zwirki i Wigury St., 02-091 Warsaw, Poland, e-mail: pmiskiewicz@wum.edu.pl

Submitted: 9.05.2018; Accepted: 11.05.2018 
sensitive and specific [12]. Third-generation assays with improved sensitivities, using M22 human monoclonal thyroid-stimulating antibodies as a competitor against the purified porcine TSH-R, were the first to be introduced to automatic immunochemical analysers [13]. To date, they are widely applied in clinical practice; however, they do not differentiate between TRAB functional types [14].

Recently, the novel fully automated IMA for TRAB measurements - Immulite 2000 Thyroid-Stimulating Immunoglobulins assay (Siemens Healthcare Diagnostics, Llanberis, UK) - has been made available $[15,16]$. It employs a pair of recombinant human TSH-Rs in a bridging format and utilises capture and signal chimeric receptors [17]. Studies on GD patients, which compared the Immulite assay with third-generation TRAB IMA, have confirmed its high specificity and sensitivity $[15,16]$. However, to the best of our knowledge, there is still no report evaluating its clinical utility in patients with GO.

Thus, we wanted to assess the clinical performance of Immulite assay in GO patients treated with ivGCs. Previous studies comparing various TRAB assays showed significant inter-method variability in TRAB measurements [18] and the difference in correlation of TRAB with GO outcome, depending on the applied test [19]. Therefore, we compared the TRAB measurements with Immulite assay (TRAB Immulite) and third-generation TRAB assay (TRAB Cobas) in the serum of patients with active, moderate-to-severe GO before, during, and at the end of the treatment with methylprednisolone (MP) pulse therapy. Additionally, we evaluated association of both immunoassays with clinical signs of GO and the final outcome of the treatment.

\section{Material and methods}

\section{Patients}

The study consisted of 40 Graves' disease patients with active, moderate-to-severe GO, who were treated with high doses of MP in the Department of Internal Diseases and Endocrinology at the Medical University of Warsaw in the period 2012-2017. The activity and severity of the eye disease were assessed according to the standardised criteria of the European Group on Graves Orbitopathy (EUGOGO). GO was classified as active if at least three of the seven items of the Clinical Activity Score (CAS) were present. All patients presented clinical and laboratory euthyroidism for at least two months, with appropriate treatment. Patients under glucocorticoid therapy in the last six months and with previous immunosuppressive treatment for GO were excluded from the study.

\section{Clinical assessment and treatment schedule}

Enrolled patients received intravenous MP pulse treatment in accordance with the EUGOGO protocol: $0.5 \mathrm{~g}$ MP once a week for six weeks and then $0.25 \mathrm{~g}$ once a week for another six weeks (cumulative dose MP: $4.5 \mathrm{~g}$ ). Initial data collected on patients included demographics, smoking habits, concomitant diseases, duration of GO and GD, and current and previous therapy of Graves' hyperthyroidism. At enrolment, thyroid ultrasound was performed in all patients, with measurement of the thyroid volume in millilitres using the ellipsoid formula. Patients underwent clinical and laboratory assessment at the following three time points: 1) directly before the beginning of treatment ( $1^{\text {st }}$ pulse $\left.), 2\right)$ in the middle of the treatment $\left(6^{\text {th }}\right.$ pulse $)$, and 3 ) after the last pulse of MP (12 $12^{\text {th }}$ pulse). Clinical evaluation included the following items: CAS features (spontaneous retrobulbar pain, pain on attempted upward or downward gaze, redness of eyelids, redness of conjunctiva, swelling of caruncle or plica, swelling of eyelids, and chemosis), lid width in mm, exophthalmos, and diplopia. The effectiveness of the treatment was evaluated after the $12^{\text {th }}$ MP pulse, and it was classified as: improvement, deterioration, or no change [20]. The study was approved by the local Bioethics Committee, and patients' informed consent was obtained.

\section{Blood sampling and assays}

Fasting blood samples were taken from a vein in the morning (7.00-9.00 a.m.) before each pulse and centrifuged. Serum was divided into two parts. The first one was sent to the Central Laboratory of the Public Central Teaching Clinical Hospital of the Medical University of Warsaw for TRAB measurement with third-generation assay and routine analysis of other parameters: TSH, free triiodothyronine (fT3), free thyroxine (fT4), thyroid peroxidase antibodies (ATPO), and thyroglobulin antibodies (ATG) level. The second part of the serum was divided into several aliquots and stored at $-80^{\circ} \mathrm{C}$. Subsequently, all samples were assessed at the same time, using TRAB Immulite assay in the Department of Laboratory Medicine and Clinical Immunology of Developmental Age of the Public Paediatric Teaching Clinical Hospital of the Medical University of Warsaw.

Immulite 2000 Thyroid-Stimulating Immunoglobulins assay (Siemens Healthcare Diagnostics, Llanberis, UK) is an automated chemiluminescence IMA with the cut-off of $0.55 \mathrm{IU} / \mathrm{l}$ and the range of referential values of 0.10-40 IU/l. Based on the manufacturer's material, the sensitivity and specificity of the Immulite assay are $98.3 \%$ and $99.7 \%$, respectively.

The level of all other serum analytes was measured using electro-chemiluminescence IMAs on a Cobas 411 automatic analyser (Roche Diagnostics, Basel, Switzerland). The cut-off and measuring range of TRAB was 1.75 IU/l and 0.30-40 IU/l, respectively. The sensitivity and specificity of the test was evaluated by the manufacturer as $97 \%$ and $99.1 \%$, respectively. TSH, fT3, and fT4 reference ranges were 0.27 to $4.2 \mathrm{mU} / \mathrm{l}, 3.1$ to $6.8 \mathrm{pmol} / \mathrm{l}$, and 12 to 
$22 \mathrm{pmol} / \mathrm{l}$, respectively, and ATPO and ATG cut-off were $34 \mathrm{IU} / \mathrm{ml}$ and $115 \mathrm{IU} / \mathrm{ml}$, respectively.

\section{Data analysis and statistics}

Statistical analysis was performed by Medcalc version 17.8.6 (MedCalc Software, Belgium). All demographic, clinical, and laboratory characteristics were presented as median with percentiles (25.75) or mean with confidence interval (CI) for continuous variables and absolute number with prevalence of dichotomous variables. In the laboratory findings values lower than the limit of quantitation (LoQ) were considered equal to LoQ for statistical purposes. Summary statistics were produced according to response status at week 12, classified as "responder" by improvement or "non-responder" by lack of improvement or deterioration. Groups were statistically compared using Mann-Whitney $\mathrm{U}$ test (metric variables), or chi-square test (dichotomous variables). In order to assess whether the change occurred between the $1^{\text {st }}$ and $12^{\text {th }}$ pulse, the Wilcoxon signed rank test was applied. The correlations of the serum TRAB Immulite and TRAB Cobas concentration with clinical variables were examined via Spearman correlation analysis. A $p$-value $\leq 0.05$ was considered for statistical significance. Correlation and agreement between TRAB Immulite and Cobas assays were assessed means of the Passing and Bablok regression analyses and Bland-Altman plots, respectively.

\section{Results}

The baseline characteristics of 40 patients included in the study are shown in Table 1. All patients completed the scheduled treatment course without any significant breaks. 26 of 40 patients $(65 \%)$ responded to MP treatment, and none of the patients' condition deteriorated. We observed no statistically significant difference between responders and non-responders, as shown in Table 1.

TRAB Immulite and TRAB Cobas were both positive in all 40 patients at the beginning of treatment. There was

Table 1. Demographic, clinical and laboratory data of patients included in the study, with characteristics of responders vs. non-responders to GO treatment. Data are expressed as absolute numbers of patients (percentage of all) or median $\left(25^{\text {th }} ; 75^{\text {th }}\right.$ percentiles $)$

\begin{tabular}{|c|c|c|c|c|}
\hline Characteristic & $\begin{array}{l}\text { All patients } \\
\qquad N=\mathbf{4 0}\end{array}$ & $\begin{array}{c}\text { Responder } \\
n=26(65 \%)\end{array}$ & $\begin{array}{c}\text { Non-responder } \\
n=14(35 \%)\end{array}$ & $p$-value \\
\hline Female & $29(72.5)$ & $18(69.2)$ & $11(78.6)$ & 0.53 \\
\hline Age (years) & $54.5(45 ; 59)$ & $55(45 ; 59)$ & $49.5(43 ; 60)$ & 0.95 \\
\hline Current smoker & $20(50)$ & $12(46.2)$ & $8(57.1)$ & 0.51 \\
\hline Never smoked & $9(22.5)$ & $6(23.1)$ & $3(21.4)$ & 0.91 \\
\hline GD duration (months) & $24(6 ; 33)$ & $24(9 ; 84)$ & $12(7 ; 59)$ & 0.92 \\
\hline Radioiodine therapy & $9(22.5)$ & $5(19,2)$ & $4(28,6)$ & 0.51 \\
\hline Thyroidectomy & $3(7.5)$ & $2(7.7)$ & $1(7.1)$ & 0.95 \\
\hline GO duration (weeks) & $36(19 ; 49)$ & $27(17 ; 41)$ & $42(32 ; 85)$ & 0.11 \\
\hline Double vision & $35(87.5)$ & $23(88.5)$ & $12(85.7)$ & 0.80 \\
\hline Constant diplopia & $19(47.5)$ & $12(46.2)$ & $7(50)$ & 0.82 \\
\hline Proptosis (mm) & $22(20 ; 24)$ & $22(20 ; 25)$ & $21(20 ; 24)$ & 0.53 \\
\hline CAS & $4(3 ; 5)$ & $4(3 ; 5)$ & $3(3 ; 4)$ & 0.09 \\
\hline Thyroid volume (ml) & $17(9 ; 27)$ & $24(18 ; 28)$ & $17(9 ; 22)$ & 0.98 \\
\hline TRAB Cobas (IU/l) $[<1.75]$ & $6.38(2.7 ; 13.5)$ & $6.38(2.8 ; 9.8)$ & $5.93(2.7 ; 26.9)$ & 0.34 \\
\hline TRAB Immulite (IU/l) $[<0.55]$ & $4.25(1.9 ; 8.2)$ & $4.55(2.2 ; 7.3)$ & $3.60(1.5 ; 14)$ & 0.56 \\
\hline ATPO (IU/ml) $[<34]$ & $191(45 ; 342)$ & $191(39 ; 345)$ & $173(50 ; 341)$ & 0.83 \\
\hline $\operatorname{ATG}(\mathrm{IU} / \mathrm{ml})[<115]$ & $69(13 ; 295)$ & $30(11 ; 232)$ & $110(15 ; 503)$ & 0.21 \\
\hline $\mathrm{TSH}(\mu \mathrm{IU} / \mathrm{ml})[0.27-4.2]$ & $1.3(0.4 ; 2.4)$ & $1.3(0.5 ; 2.1)$ & $1.1(0.2 ; 3.6)$ & 0.79 \\
\hline fT4 (pmol/l) [12-22] & $15.5(14.2 ; 18.7)$ & $15.1(13.5 ; 18.7)$ & $16.2(14.6 ; 18.7)$ & 0.57 \\
\hline fT3 (pmol/1) [3.1-6.8] & $4.8(4.1 ; 5.4)$ & $4.7(4 ; 5.3)$ & $5(4.1 ; 5.5)$ & 0.50 \\
\hline
\end{tabular}



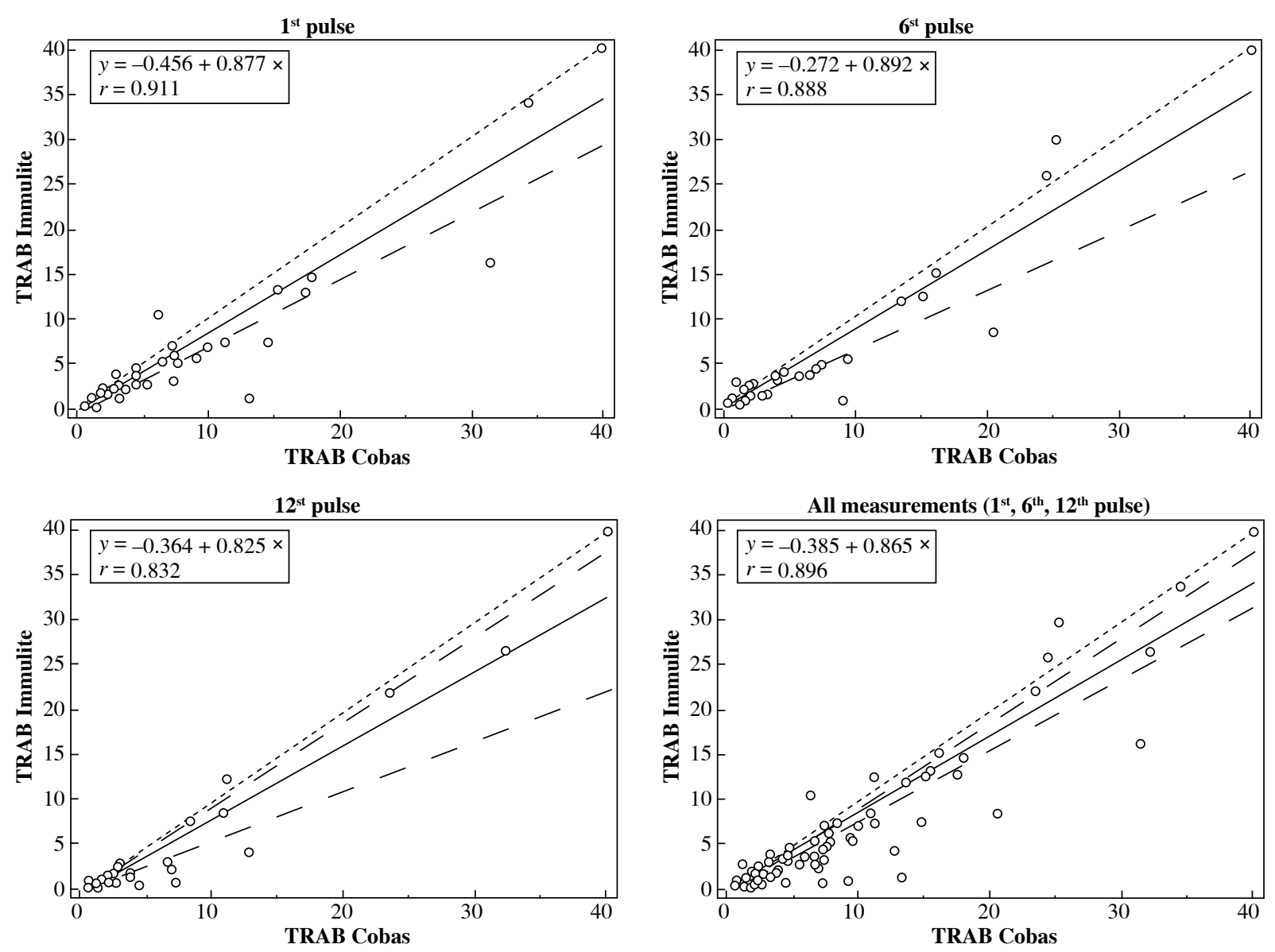

—-Solid line - regression line -. - Grey dashed lines - 95\% confidence intervals (CI) for the regression line .... Dotted line - identity line

Fig. 1. Passing and Bablok regression analysis of TSH receptor antibodies level assessed with Immulite assay (TRAB Immulite) vs. TSH receptor antibodies level assessed with Cobas assay (TRAB Cobas) at $1^{\text {st }}, 6^{\text {th }}, 12^{\text {th }}$, and during all pulses

a strong correlation between TRAB Immulite and TRAB Cobas levels in all measurements during the treatment $\left(r=0.911, r=0.888, r=0.832\right.$ at $1^{\text {st }}, 6^{\text {th }}$, and $12^{\text {th }}$ pulse, respectively), but Passing and Bablok regression analysis showed acceptable agreement only in measurements at $1^{\text {st }}$ and $6^{\text {th }}$ pulse (Fig. 1). Analysis of measurements at $12^{\text {th }}$ pulse and all measurements together revealed no acceptable agreement between both methods. The mean difference between TRAB Immulite and TRAB Cobas was $-1.5 \mathrm{IU} / 1(p<0.0001)$ (Fig. 2). The decrease of both TRAB Immulite (mean value at $1^{\text {st }}$ pulse: $8.99 \mathrm{IU} / 1$; $6^{\text {th }}$ pulse: $6.19 \mathrm{IU} / 1 ; 12^{\text {th }}$ pulse: $3.88 \mathrm{IU} / \mathrm{l}$ ) and TRAB Cobas (mean value at $1^{\text {st }}$ pulse: $10.88 \mathrm{IU} / 1 ; 6^{\text {th }}$ pulse: $7.16 \mathrm{IU} / 1$; $12^{\text {th }}$ pulse: $5.26 \mathrm{IU} / \mathrm{l}$ ) levels in serum, before and in the middle of the treatment, as well as before and after the treatment, was statistically significant $(p<0.0001)$ (Fig. 3). Median values of TRAB Immulite and TRAB Cobas levels were without difference between responders and non-responders in all points of evaluation. (Fig. 4).
Neither of the TRAB baseline levels correlated with activity or severity features of GO and were unrelated to diplopia, thyroid volume, and duration of GO and GD. Assessing results from each pulse during the treatment, a correlation was observed between both TRABs levels and GO activity (Fig. 5). Comparing the coefficients of correlation for TRAB Immulite and TRAB Cobas, there was no significant difference $(r=0.27, p=0.004 ; r=0.29$, $p=0.001$, respectively).

\section{Discussion}

Numerous experimental studies suggest the role of TSH-R as the main antigen in GO [21] and thus TRAB as a mediator of metabolic changes in orbital tissues leading to inflammation and adipogenesis in the orbit [22]. Some clinical observations support this theory reporting that both TRAB and TSAB levels correlate with GO severity and clinical activity $[6,7,23,24]$. However, other studies do not 

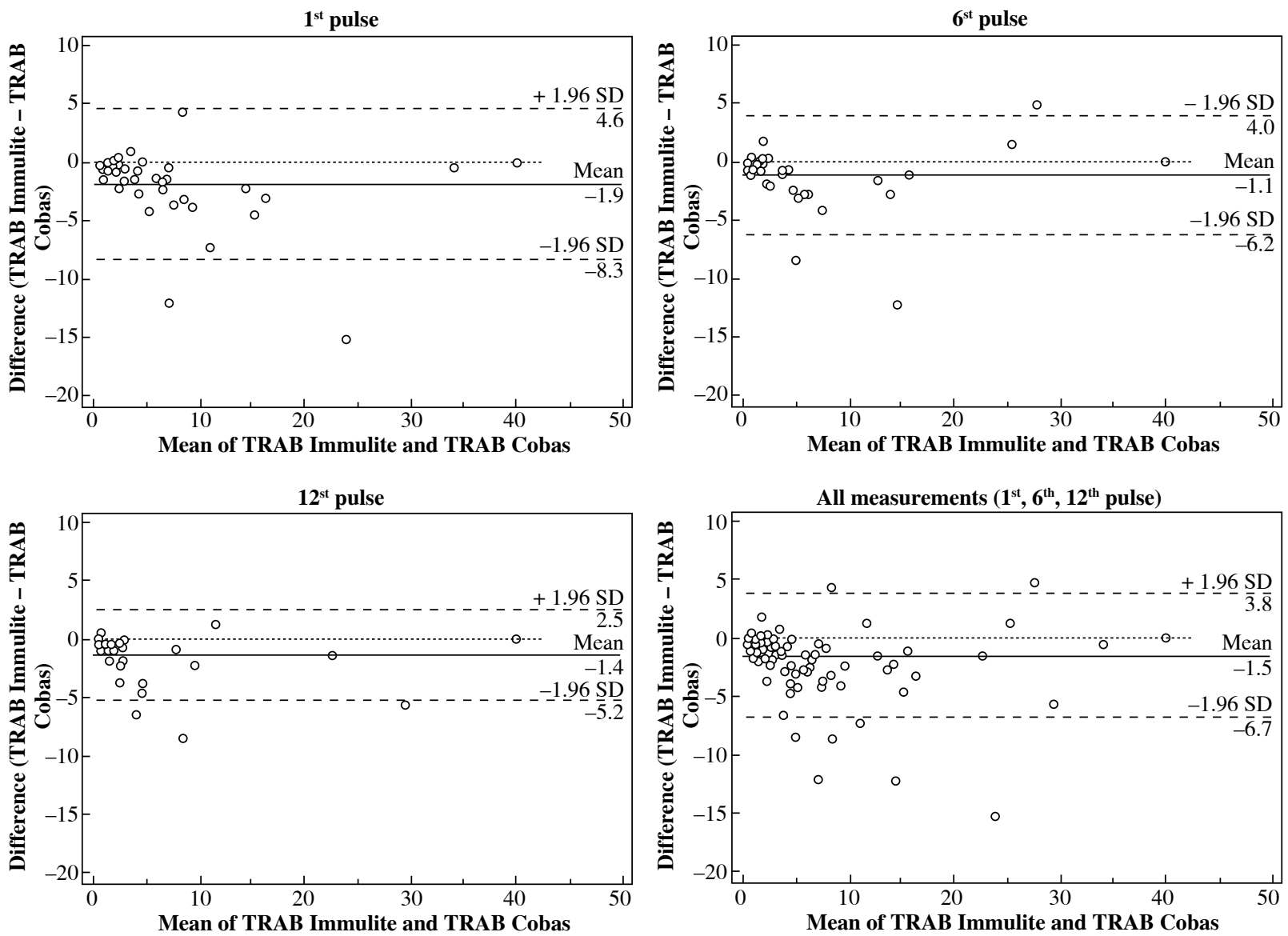

_ Solid line - mean difference _ _ - Dashed lines - standard deviation (SD) ... Dotted line - difference of zero

Fig. 2. The Bland-Altman plot analysis of the difference between TSH receptor antibodies level assessed with Immulite assay (TRAB Immulite) versus TSH receptor antibodies level assessed with Cobas assay (TRAB Cobas) in serum at $1^{\text {st }}$, $6^{\text {th }}, 12^{\text {th }}$, and all pulses

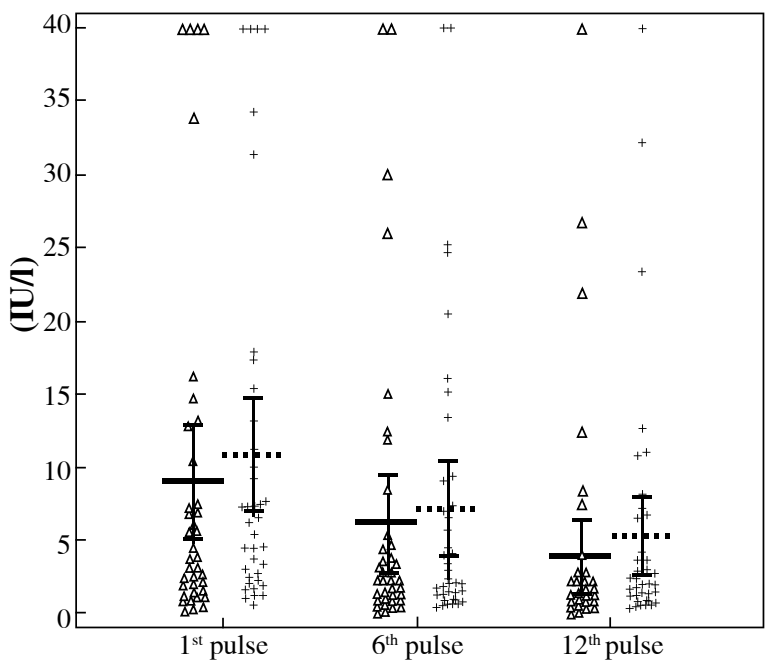

$$
\begin{aligned}
& p<0.0001 \\
& \Delta \text { TRAB (Immulite, Siemens) } \\
& + \text { TRAB (Cobas, Roche) }
\end{aligned}
$$

Fig. 3. TSH receptor antibodies level assessed with Immulite assay (TRAB Immulite) and TSH receptor antibodies level assessed with Cobas assay (TRAB Cobas) in serum measured at three time points during the treatment. Diagram shows the mean value (long cross line) with confidence intervals (short cross line). Bullets represent individual antibodies level 


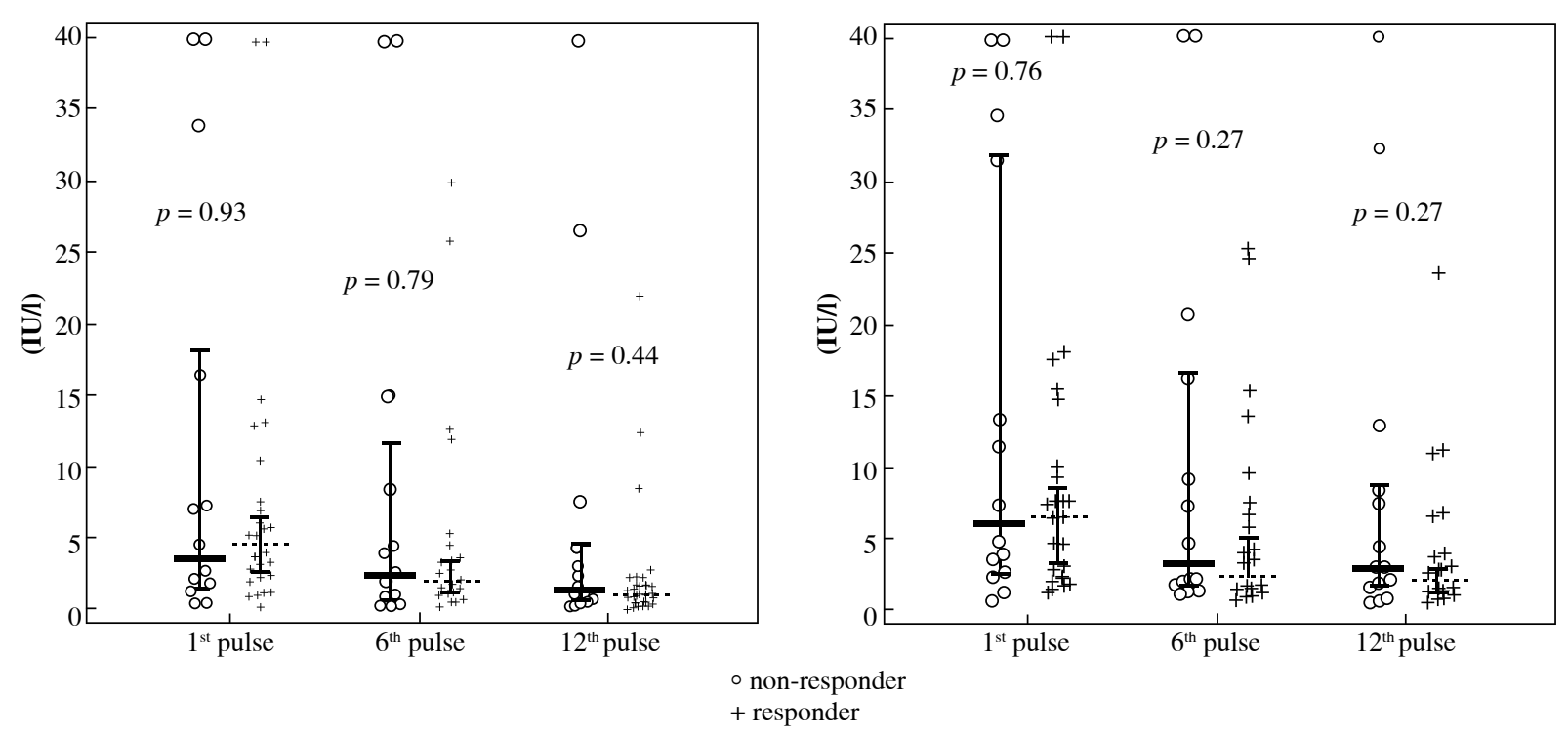

Fig. 4. Responders and non-responders TSH receptor antibodies level assessed with Immulite assay (TRAB Immulite) and TSH receptor antibodies level assessed with Cobas assay (TRAB Cobas) in serum measured at three time points during the treatment. Diagram shows the median value (long cross line) with confidence intervals (short cross line). Bullets represent individual antibodies level
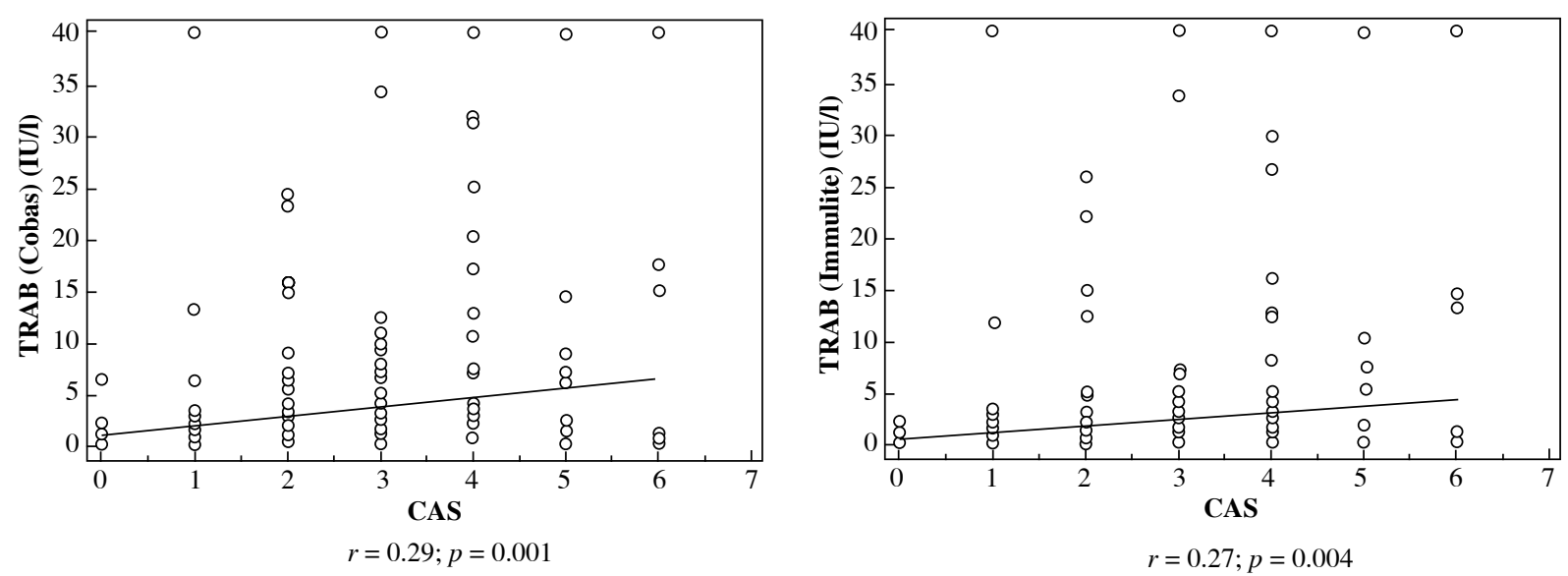

Fig. 5. Correlation of the TSH receptor antibodies assessed with Immulite assay (TRAB Immulite) and TSH receptor antibodies assessed with Cobas assay (TRAB Cobas) with GO activity evaluated as Clinical Activity Score (CAS) during the intravenous glucocorticoid pulse therapy. A single dot represents a single sample

confirm this association indicating the correlation of stimulating TSH-R antibodies (TSAB) but not TRAB with GO outcome [25-27]. The possible reason for this discrepancy may be the difference between applied TRAB assays. Massart et al. revealed significant inter-method variability, even between two similar third-generation TRAB assays using a human monoclonal TSH-R-stimulating autoantibody M22 for competitive binding [18]. Moreover, in the study of Lytton et al. association of TRAB level with GO outcome was evaluated as very weak or moderate depending on the ap- plied assay (TRAK Human radioimmunoassay and ELISA Kit, respectively) [19]. Despite the different technologies of examined assays and the specificity of the Immulite assay for TSAB declared by the producer [28], in our study we did not observe any significant discrepancy between Cobas and Immulite tests. Initially, TRAB Immulite and TRAB Cobas levels were not associated with activity or signs and symptoms indicating GO severity. However, examining results from the whole time of treatment, the weak correlation between both antibodies and clinical activity was observed, 
with no significant difference between both TRABs $(r=$ $0.29, p=0.001 ; r=0.27, p=0.004$ respectively). Moreover, as expected, we observed a significant decrease of both antibodies levels in serum during the treatment.

Immulite assay is based on the chimeric receptor that, according to the producer, specifically binds TSAB but not TSH-R blocking antibodies (TBAB) [17]. This is due to genetic modification of native TSH-R and the replacement of TBAB epitope by lutropin/choriogonadotropin (LH/CG) one. Current studies report that diagnostic performance of Immulite in GD patients is at least comparable to third-generation TRAB assay underlining its greater accuracy $[15,16]$. In our study we observed strong correlation between both assays and significant agreement between two methods before the ivGCs treatment. Diana et al. suggested that the Immulite assay cannot differentiate between TSAB and TBAB because all hypothyroid patients with high levels of TBAB included in her study were positive in the Immulite assay [29]. Our results may support this observation because the correlation between Immulite and Elecsys assays was significantly stronger than the correlation between TSAB and TRAB levels in previous study from our institution ( $r=0.91 ; r=0.33$, respectively) [30].

In our study, the final outcome of ivGCs was not associated with initial or final TRAB levels, which may suggest that it cannot be used as a prognostic marker in GO treatment, which would support some previous reports [31, $32]$ but is discordant with others [7, 8, 23]. Jang et al. noticed that various GO and GD durations and different status of hyperthyroidism treatment have a significant influence on TRAB levels and may cause discordance between the results [23]. Indeed, 28 out of 40 patients from our study group received antithyroid drug or block and replace therapy. The dynamic changes in TRAB levels during the first months of the treatment [33] could cause high variability in antibody levels every week and hence make the association with GO outcome difficult to interpret.

Our study has significant limitations. The small patient cohort $(n=40)$ included only euthyroid patients with active moderate-to severe GO, without patients with inactive, mild, or sight-threatening GO. This may have contributed to the lack of correlation between antibodies level and GO outcome. It may be supported by the fact that we found a correlation of TRAB with GO activity when we assessed the measurements from each pulse during the treatment, i.e. when the activity and severity of GO was more heterogeneous. Moreover, numerous reports with larger patient cohorts investigated TRAB correlation with GO outcome before. However, to the best of our knowledge, our study is the first one examining Immulite assay utility in patients with GO and during ivGCs treatment. Nevertheless, further investigation of the clinical characteristics of Immulite assay is required to prove its specificity as declared by manufacturer.

\section{Conclusions}

In conclusion, we found a close correlation between TRAB Immulite and TRAB Cobas and a similar clinical utility in patients with moderate-to-severe GO treated with ivGCs.

\section{Acknowledgments}

We thank Dorota Adamczyk for her excellent technical assistance.

The authors declare no conflict of interest.

\section{References}

1. Bartalena L, Fatourechi V (2014): Extrathyroidal manifestations of Graves' disease: a 2014 update. J Endocrinol Invest 37: 691 .

2. Bartalena L, Baldeschi L, Boboridis K, et al. (2016): The 2016 European Thyroid Association/European Group on Graves' Orbitopathy Guidelines for the Management of Graves' Orbitopathy. Eur Thyroid J 5: 9-26.

3. Tanda ML, Piantanida E, Liparulo L, et al. (2013): Prevalence and natural history of Graves' orbitopathy in a large series of patients with newly diagnosed graves' hyperthyroidism seen at a single center. J Clin Endocrinol Metab 98: 1443-1449.

4. Bartalena L, Krassas GE, Wiersinga W, et al. (2012): Efficacy and safety of three different cumulative doses of intravenous methylprednisolone for moderate to severe and active Graves' orbitopathy. J Clin Endocrinol Metab 97: 4454-4463.

5. Estcourt S, Hickey J, Perros P, et al. (2009): The patient experience of services for thyroid eye disease in the United Kingdom: results of a nationwide survey. Eur J Endocrinol 161: 483-487.

6. Gerding MN, van der Meer JW, Broenink M, et al. (2000): Association of thyrotrophin receptor antibodies with the clinical features of Graves' ophthalmopathy. Clin Endocrinol (Oxf) 52: 267-271.

7. Eckstein AK, Plicht M, Lax H, et al. (2006): Thyrotropin receptor autoantibodies are independent risk factors for Graves' ophthalmopathy and help to predict severity and outcome of the disease. J Clin Endocrinol Metab 91: 3464-3470.

8. Eckstein AK, Plicht M, Lax H, et al. (2004): Clinical results of anti-inflammatory therapy in Graves' ophthalmopathy and association with thyroidal autoantibodies. Clin Endocrinol (Oxf) 61: 612-618.

9. Kahaly GJ (2015): Bioassays for TSH receptor antibodies: quo vadis? Eur Thyroid J 4: 3-5.

10. Zophel K, Roggenbuck D, Wunderlich G, et al. (2010): Continuously increasing sensitivity over three generations of TSH receptor autoantibody assays. Horm Metab Res 42: 900-902.

11. Smith BR, Hall R (1974): The thyroid stimulating hormone receptor and thyroid stimulating antibodies. J Endocrinol 63: $59-60$.

12. Costagliola S, Morgenthaler NG, Hoermann R, et al. (1999): Second generation assay for thyrotropin receptor antibodies has superior diagnostic sensitivity for Graves' disease. J Clin Endocrinol Metab 84: 90-97. 
13. Smith BR, Bolton J, Young S, et al. (2004): A new assay for thyrotropin receptor autoantibodies. Thyroid 14: 830-835.

14. Barbesino G, Tomer Y (2013): Clinical utility of TSH receptor antibodies. J Clin Endocrinol Metab 98: 2247-2255.

15. Tozzoli R, D'Aurizio F, Villalta D, Giovanella L (2017): Evaluation of the first fully automated immunoassay method for the measurement of stimulating TSH receptor autoantibodies in Graves' disease. Clin Chem Lab Med 55: 58-64.

16. Autilio C, Morelli R, Locantore P, et al. (2018): Stimulating TSH receptor autoantibodies immunoassay: analytical evaluation and clinical performance in Graves' disease. Ann Clin Biochem 55: 172-177.

17. Frank CU, Braeth S, Dietrich JW, et al. (2015): Bridge Technology with TSH Receptor Chimera for Sensitive Direct Detection of TSH Receptor Antibodies Causing Graves' Disease: Analytical and Clinical Evaluation. Horm Metab Res 47: 880-888.

18. Massart C, Sapin R, Gibassier J, at al. (2009): Intermethod variability in TSH- receptor antibody measurement: implications for the diagnosis of Graves' disease and for the follow up of Graves' ophthalmopathy. Clin Chem 55: 183-186.

19. Lytton SD, Ponto KA, Kanitz M, et al. (2010): A novel thyroid stimulating immunoglobulin bioassay is a functional indicator of activity and severity of Graves' orbitopathy. J Clin Endocrinol Metab 95: 2123-2131.

20. Bartalena L, Veronesi G, Krassas GE, et al. (2017): Does early response to intravenous glucocorticoids predict the final outcome in patients with moderate-to-severe and active Graves' orbitopathy? J Endocrinol Invest 40: 547-553.

21. Khoo TK, Bahn RS (2007): Pathogenesis of Graves' Ophthalmopathy: The Role of Autoantibodies. Thyroid 17: 1013-1018.

22. Kumar S, Nadeem S, Stan MN, et al. (2011): A stimulatory TSH receptor antibody enhances adipogenesis via phosphoinositide 3-kinase activation in orbital preadipocytes from patients with Graves' ophthalmopathy. J Mol Endocrinol 46: 155-163.

23. Jang SY, Shin DY, Lee EJ, et al. (2013): Relevance of TSH-receptor antibody levels in predicting disease course in Graves' orbitopathy: comparison of the third-generation TBII assay and Mc4-TSI bioassay. Eye (Lond) 27: 964-971.

24. Ponto KA, Kanitz M, Olivo PD, et al. (2011): Clinical relevance of thyroid-stimulating immunoglobulins in Graves' ophthalmopathy. Ophthalmology 118: 2279-2285.

25. Noh JY, Hamada N, Inoue Y, et al. (2000): Thyroidstimulating antibody is related to Graves' ophthalmopathy, but thyrotropin-binding inhibitor immunoglobulin is related to hyperthyroidism in patients with Graves' disease. Thyroid 10: 809-813.

26. Khoo DH, Ho SC, Seah LL, et al. (1999): The combination of absent thyroid peroxidase antibodies and high thyroid-stimulating immunoglobulin levels in Graves' disease identifies a group at markedly increased risk of ophthalmopathy. Thyroid 9: 1175-1180.

27. Stan MN, Bahn RS (2010): Risk Factors for Development or Deterioration of Graves' Ophthalmopathy. Thyroid 20: 777-783.

28. Bitcon V, Donnelly J, Kiaei D (2016): Sensitivity of assays for TSH-receptor antibodies. J Endocrinol Invest 39: 1195-1196.

29. Diana T, Wuster C, Kanitz M, Kahaly GJ (2016): Highly variable sensitivity of five binding and two bio-assays for TSH-receptor antibodies. J Endocrinol Invest 39: 1159-1165.
30. Jonas M, Ambroziak U, Nauman J (2006): Correlation between thyroid stimulating immunoglobulins and thyrotropin binding inhibitory immunoglobulins levels in patients with Graves' disease. Endokrynol Pol 57: 23-30.

31. Leo M, Mautone T, Ionni I, et al. (2016): Variables Affecting the Long-Term Outcome of Graves Orbitopathy Following High-Dose Intravenous Glucocorticoid Pulse Therapy in Patients Not Treated with Orbital Radiotherapy. Endocr Pract 22: $1177-1186$.

32. Jagiello-Korzeniowska A, Sokolowski A, KrzentowskaKorek A, et al. (2016): The efficacy of immunosuppressive treatment of Graves' orbitopathy is not affected by previous anti-thyroid drugs or by radioiodine therapy of Graves' disease. Endokrynol Pol 67: 554-561.

33. Laurberg P, Wallin G, Tallstedt L, et al. (2008): TSH-receptor autoimmunity in Graves' disease after therapy with antithyroid drugs, surgery, or radioiodine: a 5-year prospective randomized study. Eur J Endocrinol 158: 69-75. 